\title{
Identification of One BOCR Mutation and Five NF1 Mutations in Male Patients with Neurofibromatosis Type 1 and Congenital Pseudarthrosis of the Tibia
}

\author{
Zhifan Zhou ${ }^{1,2,3}$, Yong Zeng ${ }^{4}$, Xinchun $\mathrm{Wu}^{4}$, Fei $\mathrm{Li}^{4}$, Xiaoyan $\mathrm{Liu}^{1,2,3}$, Lu Zhou ${ }^{1,2,3}$, Lihua Hou ${ }^{1,2,3}$, \\ Silong Sun ${ }^{5}$, Lu Shen ${ }^{1,2,3}$,Zhangyuan Lin ${ }^{4, *}$ \\ ${ }^{1}$ Department of Neurology, Xiangya Hospital, Central South University, Changsha 410008, China \\ ${ }^{2}$ Hunan Province Key Laboratory in Neurodegenerative Disorders, Central South University, Changsha \\ 410008, China \\ ${ }^{3}$ State Key Laboratory of Medical Genetics, Changsha 410008, China \\ ${ }^{4}$ Department of Orthopaedics, Xiangya Hospital, Central South University, \#87 Xiangya Road, Changsha \\ 410008, China \\ ${ }^{5}$ BGI-Shenzhen, Shenzhen, Guangdong Province, 518083, China \\ *Correspondence: linzhangyuan2505@gmail.com; TEL:+86-13907483019; Fax: +86-731-84327332
}

\begin{abstract}
Neurofibromatosis type1 (NF1) is an autosomal dominant disorder caused by mutations in the NF1gene. Although congenital pseudarthrosis of the tibia (CPT) has frequently been associated with NF1, the underlying molecular mechanism of CPT in these NF1 patients is yet ill-understood. The aim of the present study was to detect NF1 mutations from genomic DNA and to harbor variants associated with CPT in NF1 patients. Whole-exome sequencing was first carried out with samples from two patients with CPT in one NF1 family, and a novel mutation c.2324A $>\mathrm{G}$ (p.E775G) in NF1 gene was identified. Additionally, a missense variant c.455C $>$ T (p.P152L) in BCOR gene completely co-segregated with the CPT phenotype within this family. Subsequently, NF1 and NF2 genes in four other unrelated patients with both NF1 and CPT were screened using targeted sequencing. Four mutations in NF1 gene, including two known mutations (c.2288T >C/p.L763P, c.574 $\mathrm{C}>\mathrm{T} / \mathrm{p} . \mathrm{R} 192^{*}$ ) and two novel mutations (c.768delT/p.F256Lfs*25, c.2229_2230delTG/ p.V744Qfs*23) were detected. Further study confirmed that CPT was present in NF1 families, and NF1 mutations were closely associated with these complex phenotypes. Moreover, the data from the current study indicated that male gender might be a susceptibility factor for CPT in NF1. Therefore, we speculated that $B C O R$ variants might be related to CPT phenotype among male NF1 patients.
\end{abstract}

Keywords: neurofibromatosis type 1; congenital pseudarthrosis of the tibia; whole-exome sequencing; targeted sequencing; $B C O R$

\section{Introduction}

Neurofibromatosis type 1 (NF1; OMIM\#162200) is one of the most common autosomal dominant genetic disorder affecting multiple organ systems, occurring with a worldwide incidence of 1 in 2,500 to 3,000 individuals [1, 2]. It is caused by mutations in the NF1 gene located at chromosome 17q11.2, which encodes for the protein neurofibromin and is involved in the regulation of the RAS/mitogen-activated protein kinases (MAPK) pathway. A clinical diagnosis of NF1 is typically based on distinctive phenotypic features, such as café-au-lait spots, axillary freckling, optic gliomas, neurofibromas, Lisch nodules, and bone lesions. Bone lesions in NF1 patients include scoliosis, sphenoid wing dysplasia, and long-bone dysplasia. Long-bone dysplasia is almost always unilateral and presents as anterolateral bowing that may progress to fracture and nonunion (i.e., pseudarthrosis). The long bone dysplasia typically involves the tibia; however, not all individuals with tibial dysplasia progress to pseudarthrosis. Approximately 3-4\% patients with NF1 presented 
clinical and pathological evidence of tibial pseudarthrosis [3].

Congenital pseudarthrosis of the tibia (CPT) is an extremely rare disease with an estimated frequency of 1/140,000 births [4]. The molecular causes of CPT are yet unknown. As one of the long bone dysplasia, it is characterized by anterolateral bowing of tibia and recurrent pathological fractures of the tibia or fibula during early childhood. Consecutive bone healing is usually insufficient, resulting in the development of pseudarthrosis with fibrous tissue. This fibrous tissue in the region of pseudarthrosis is also known as fibrous hamartoma [5], in continuity with abnormal periosteal thickening observed in histological studies [6]. A majority of the cells of this hamartoma have been identified as fibroblasts [7]. A certain amount of fibrocartilage and hyaline cartilage [8] is also associated with the fibrous tissue, as well as, several areas of endochondral ossification. The composition of the pseudarthrotic tissue does not vary significantly, irrespective of its association with NF1 [9]. Moreover, it has been reported that a large proportion of CPT patients were afflicted by NF1 (84.0\%, Cl95=69.6-98.4\%) [10], which has been higher in ratio than CPT patients without NF1.

CPT has long been associated with NF1 through the overlapping of clinical phenotypes and pathological characteristics, which indicates similar underlying genetic causes. The double inactivation of the NF1 gene is postulated as essential for the development of CPT [11]. Moreover, a wide clinical variability of CPT in the same NF1 family occurs, suggesting the possibility of additional genetic causes and/or modifiers. A variant in the PTPN11 gene (c.1658C >T/ p.T553M, rs148176616), also involved in the RAS signal transduction pathway, was identified in a patient with NF1 and CPT, along with one NF1 mutation [12]. Although the above studies have considerably advanced our understanding of the pathogenic mechanisms of patients with CPT and NF1, the genetic architecture of these complex phenotypes has not yet been completely clarified. In the present study, we first detected NF1 mutations in six Chinese Han patients with NF1 and CPT to determine whether each patient has NF1 mutations and whether these mutations play a role in the complex phenotypes. Furthermore, we explore whether variants in the other genes, as additional genetic causes, might be responsible for CPT in these NF1 patients.

\section{Results}

We studied six male patients with NF1 and CPT from five unrelated families. Two patients (A2-II:1 and A3-II:2) were sporadic cases whose first-degree relatives neither presented NF1 nor $\mathrm{CPT}$, whereas the others inherited NF1 from one of their parents. In these families (A1, A4, A5), the genetic transmission of NF1 appeared most consistent with autosomal dominant inheritance. The clinical features of all the patients were listed in Table 1. The NF1-associated dermic phenotype was age-dependent. Anterolateral bowing of the tibia was unilateral and presented at birth; however, the onset age of tibial fracture ranged from shortly after birth to 20 years (Table 1).

Analysis of the sequencing data revealed high coverage and read depths in six patients (Table 2). The initial variants were filtered out using public databases in combination with segregation analysis. We performed exome sequencing analysis in two affected individuals (A1-II:1, III:1) with CPT in the same NF1 family. A heterozygous variant (c.2324A>G/ p.E775G) in NF1 gene was identified in the pedigree, which was completely co-segregated with the disease phenotype of NF1 in the family (Figure 1A). The variant was absent in the five hundred unaffected healthy controls as assessed by Sanger sequencing. The amino acid residue (p.E775) is highly conserved in neurofibromin proteins of other species (Figure 1B). Moreover, the amino acid with a negative charge (Glu) was transformed to an uncharged amino acid (Gly), which may affect the protein structure. This mutation is a novel pathogenic mutation at genomic position chr17:29554308 (GRCh37/hg19 reference); we did not detect any NF2 rare variants. Also, any variants, except for the above described in NF1, were not identified in the fifteen other genes involved in the RAS/MAPK pathway. However, a missense variant (c.455C>T/p.P152L) in BCOR gene (chrX:39934144) was consistent with the CPT phenotype after co-segregation (Figure 1A). Two patients (A1-II:1, III:1) 
with CPT and NF1 carried the maternal allele, indicating that they were hemizygous for the variant. Although the mother (A1-I:2) of the patient (A1-II:1) was a heterozygote carrier of the variant, she was normal. On the other hand, the mother (A1-II:3) of the patient (III:1) had been diagnosed as NF1 before death; however, she did not present any clinical fractures of CPT. It was considered that she (II:3) might have the same BCOR variant and transmit it from her mother to her son, although her sample was unavailable. The variant in BCOR gene was not observed in the other available family members (Figure 1A). Additionally, this variant was not found in the ESP6500 and the 1000 Genomes Project while it was found in the Single Nucleotide Polymorphism Database (rs375342424). The allele frequency of this variant was $9.132 \times 10^{-5}$ in the ExAC database. Moreover, the missense substitution was also not detected in the five hundred unaffected control individuals, indicating the rarity of the variation in the demographic population. Pro152 was found at a highly conserved position, suggesting a critical role for this residue. (Figure1C). The rare variant was predicted to be possibly damaging by SIFT and PolyPhen2.

Subsequently, we screened for NF1 and NF2 genes in four other unrelated patients with NF1 and $\mathrm{CPT}$ by targeted sequencing. Although no variants in the NF2 gene were detected, four heterozygous variants in the NF1 gene were identified. Two of these (c.2288T>C/p.L763P, c.574 C $>$ T/p.R192*) were previously reported mutations [13-15], and the others (c.768delT/p. F256Lfs*25, c.2229_2230delTG/p.V744Qfs*23) were novel (Figure 1A). These variants were predicted as damaging by SIFT, or formed premature stop codons (PTCs) in one allele of the NF1 gene. These variants are also highly conserved in the protein neurofibromin in the other species (Figure 1B). The clinical characteristics and mutations of these patients detected in the NF1gene were summarized in Table 1. We amplified the coding region of the BCOR and PTPN11 genes using Sanger sequencing. However, no rare variants were detected in these four unrelated patients with NF1 and CPT.

\section{Discussion}

In the six Chinese patients with NF1 and CPT, we identified two missense, one nonsense, and two deletion mutations in the NF1 gene. These mutations detected using high throughput sequencing method and validated by Sanger sequencing were rarely found in large sequencing databases of controls. Two of them were "de novo" mutations, and the others originated from their parents. Similar to previous studies $[8,11,12,16]$, our results further confirmed that CPT always existed in the NF1 families and each patient with both NF1 and CPT was found harboring mutations in the NF1 gene, suggesting these mutations were closely associated with the complex phenotypes. In addition, the age of patients sustaining tibial fracture was found to be distinctly different. Age at tibial fracture ranged from 0.8 to 2 years in the patients with truncated mutations while that of tibial fracture ranged from 8 to 20 years in those with missense mutations. Patients with truncated mutations had earlier CPT onset than those with missense mutations, suggesting a correlation between the types of mutation in the NF1 gene and the age of CPT onset.

The marked variability in clinical phenotypes was observed among affected individuals from the same NF1 family [17], which was also observed in this study. Not all individuals with NF1 have CPT. All the patients with NF1 and CPT in our study were male. Thus, a male predominance was observed among these patients with complex phenotypes. Stevenson also described the phenomenon and postulated that male gender may be a susceptibility factor for CPT in NF1, through a descriptive analysis of CPT in a large cohort of NF1 patients [18].

Although the molecular pathogenesis of NF1-related tibial pseudarthrosis was poorly understood, it may partially be explained by genetic modifiers [12]. In the current study, we found a rare missense variant (c.455C $>$ T/p.P152L) in the BCOR gene completely co-segregated with the CPT phenotype in the family A1. It was rarely found in large sequencing databases of controls and was predicted on being possibly damaging, suggesting that it might be of unknown significance in the Chinese pedigree. BCOR gene is localized on chromosome $\mathrm{Xp11.4}$ and encodes the ubiquitously 
expressed BCL-6 corepressor [19]. It may specifically inhibit the gene expression when recruited to promoter regions by sequence-specific DNA-binding proteins such as BCL6 and MLLT3. This repression may be mediated at least partially by class I and II histone deacetylases [20]. Furthermore, BCL-6 corepressor, involved in repression of TFAP2A, acts as a negative regulator of osteo-dentinogenic capacity in adult stem cells [21]. FBXL11 histone demethylase function was activated by associating with BCL-6 corepressor, and FBXL11 regulated osteo-dentinogenic differentiation in MSCs [22]. BCL-6 corepressor may also participate in osteo-dentinogenic differentiation. Moreover, most mutations in the $B C O R$ gene were known to cause Lenz microphthalmia and oculofaciocardiodental syndromes (OFCD, OMIM 300166) [23]. Interestingly, these two syndromes are inherited in an X-linked pattern and comprise skeletal anomalies. Compared with these two syndromes, our patients with the BCOR variants were male with skeletal manifestations. We speculated that the probability of developing CPT might be high among these male NF1 patients with $B C O R$ variants. However, tissues from the tibial pseudarthrosis region were not collected from the two individuals with the variant. Moreover, the number of these cases in the present study was small for a clear genetic evidence to support this speculation.

\section{Experimental Section}

\section{Patients and DNA extraction}

A cohort of six male Chinese patients (mainland China) with NF1 and CPT was recruited from the orthopedics clinic of the Xiangya Hospital, Central South University, from June 2013 to June 2015. Two of these patients belonged to the same NF1 family (A1-II:1, III:1), remarkably. Each patient met standard neurofibromatosis I diagnostic criteria created by the National Institute of Health (NIH) [24]. These patients were diagnosed with CPT independently by clinical and radiological signs of dislocation by at least two well-experienced orthopedists. CPT phenotype was showed on these radiographs of five probands (Figure 2). In total, five hundred healthy Chinese individuals were recruited from the Xiangya Wellness Center as a control group. The study was approved by the Ethics Committee of the Xiangya Hospital of the Central South University in China (equivalent to an Institutional Review Board) and carried out in accordance with the approved guidelines. A written informed consent was obtained from patients or parents of sick children. Genomic DNA was extracted from peripheral blood leukocytes by standard extraction methods.

\section{Whole exome sequencing and variant discovery}

The SeqCap EZ Human Exome Library was used to enrich exonic DNA from two individuals with CPT and NF1 in the same pedigree (A1). Subsequent sequencing using HiSeq2000 (Illumina, San Diego, CA, USA) was performed to generate paired-end reads (90 bp at each end). The sequenced reads were aligned to the human genome reference (UCSC hg19) using Burrows-Wheeler Alignment (BWA) software [25]. Duplicate read removal, format conversion, and indexing were performed with Picard. The Genome Analysis Tool was used to recalibrate the base quality scores, perform local realignments around potential indels, and to call and filter the variants $[26,27]$. Subsequently, genetic variations were annotated using ANNOVAR package. The candidate variants were shared with two patients. We focused only on variants located in the splice sites or coding sequences. We also filter out the synonymous substitutions. The common variants (frequency $>1 \%$ ) were excluded by comparing the identified variants with those in the dbSNP (http://www.ncbi.nlm.nih.gov/projects/ SNP/), the NHLBI Exome Sequencing Project 6500 (ESP6500), and the 1000 Genome Project (http://www.1000genomes.org). The potential disease-causing variants were evaluated by reference tools such as SIFT predictions (http://sift.jcvi.org/) or association with an appropriate clinical syndrome. After the above filtering pipeline, rare variants shared among two patients were initially investigated including NF1 and NF2 genes. Subsequently, fifteen other genes involved in the RAS/MAPK pathway (SPRED1, RASA1, RAF1, HRAS, BRAF, KRAS, MEK1/MAP2K1, MEK2/MAP2K2, PTPN11, SOS1, PTEN, KIT, $M A P K 1 / E R K 2$, SPRED2, and TP53) were also studied. Finally, variant investigation was expanded 
to the genes responsible for inherited disorders characterized by skeletal features (SLC26A2, COMP, COL9A1, FGFR1, FGFR2, FGFR3, COL1A1, COL1A2, CRTAP, SLC35D1, ARSE, EBP, TCOF1, CLCN7, and $B C O R$ ) and genes associated with metabolism, development of bone, and proliferation and differentiation of mesenchymal stem cells (MSCs). The variant counts at each stage of filtering are summed in supplementary figure1.

\section{Targeted sequencing and Sanger sequencing}

A custom capture array (Roche Nimble Gen, Madison, WI, USA) was designed to capture two genes (NF1and NF2) known to be associated with neurofibromatosis. We examined genomic DNA samples from four clinically affected subjects with NF1 and CPT by targeted sequencing. To verify the detected NF1/ NF2 variants and candidate variants in these probands, we used Sanger sequencing to screen family members and five hundred individuals as a healthy control. Primer 5.0 was used to generate primers for the amplification of the target gene sites and related flanking sequences (NF1; RefSeq NM_001042492). We also amplified the coding region of BCOR (RefSeqNM_001123385) and PTPN11 (RefSeqNM_002834), including the flanking intronic sequences. Primer sequences were seen in supplementary table1-3.

Genomic DNAs were amplified by polymerase chain reaction (PCR) using Roche Fast Start PCR Master Mix polymerase (Roche Diagnostics Corp, USA). Sequencing reactions were performed by mixing previous PCR products and primers with ABI BigDye dye terminator cycle sequencing kits (Applied Biosystems, Foster City, CA, USA) in an ABI3730XL DNA analyzer (Applied Biosystems). Further analysis was performed using Chromas software 2.22 (Technelysium Pty Ltd, Brisbane, Australia).

\section{Conclusion}

Taken together, we identified five NF1 mutations in patients with NF1 and CPT from mainland China and speculated that BCOR variants might be related to CPT phenotype among NF1 patients. 
A

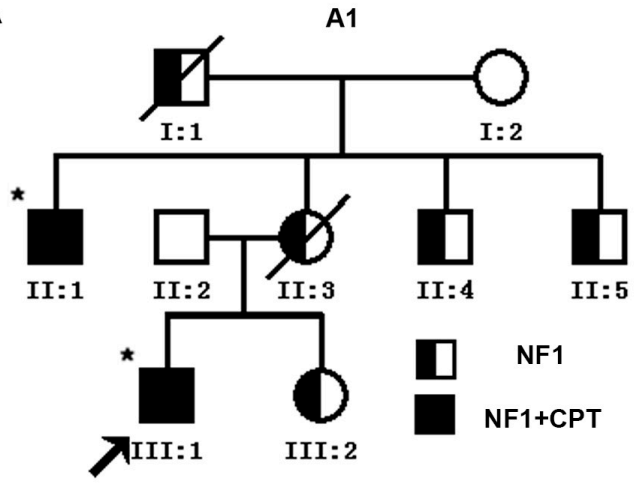

NF1 c.2324A>G/p.E775G BCOR c.445C>T/p.P152L

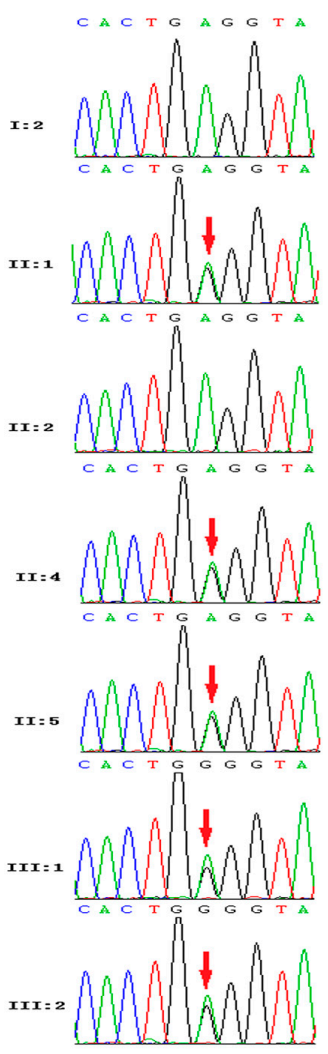

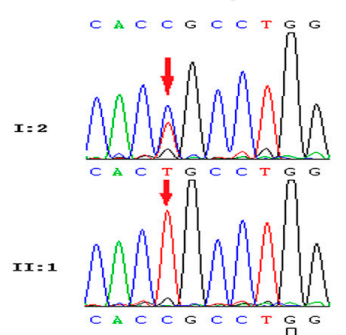
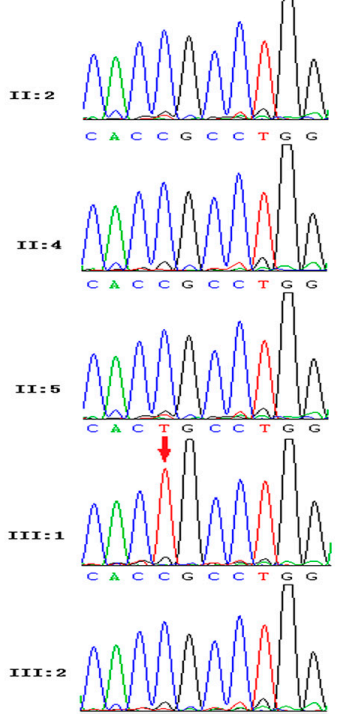

A2
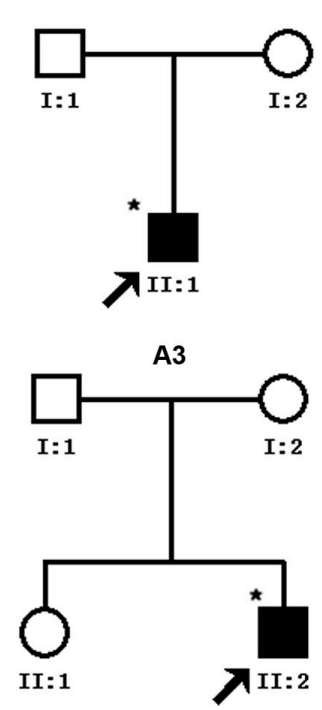

NF1 c.768delT/p. F256Lfs*25

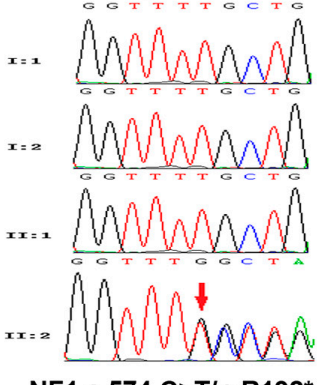

NF1 c.574 C>T/p.R192*
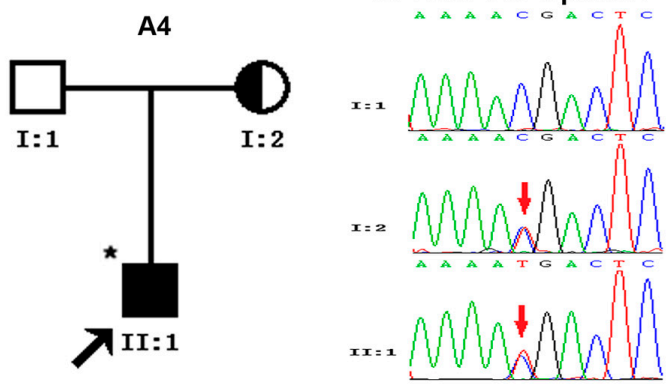

NF1 c.2229 2230delTG/p.V744Qfs 23

A5
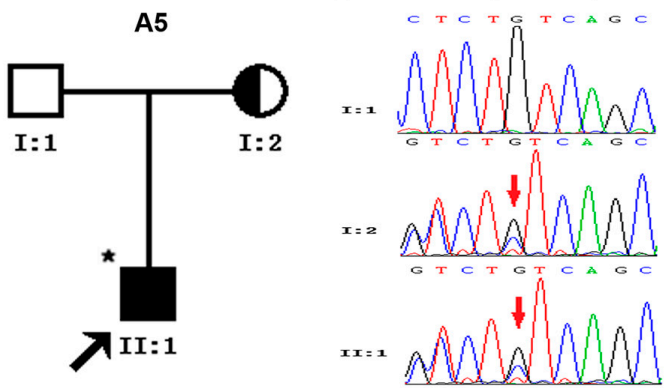

B

c.574 C>T c.768delT c.2229_2230delTG c.2288T>C c.2324A $>$ G $\begin{array}{lllll}\text { p.R192* } & \text { p. F256Lfs*25 } & \text { p.V744Qfs*23 } & \text { p.L763P } & \text { p.E775G }\end{array}$

$\begin{array}{ll}\text { Homo } & \text { KLKRLLKET_VDGFAESTK-FASVSNMMS-MALLRRIEHPTAGNTEAWEDT } \\ \text { Mus } & \text { KLKRLLKET-VDGFAESTK-FASVSNMMS-MALLRRIEHPTAGNIEAWEDT } \\ \text { Rattus } & \text { KLKRLLKET_-VDGFAESTK-FASVSNM LS-MALLRRIEHPTAGNTEAWEDT } \\ \text { Xenopus } & \text { KLKRLLQET-VDSFAESNK-FASVSNMVS-MALLRIEHPTAGNTEAWEDT } \\ \text { Macaca } & \text { KLKRLLKET-VDGFAESTK-FASVSNMMS-MALLRRIEHPTAGNTEAWEDT } \\ \text { Gallus } & \text { KLKRLLQET-VDGFAESTK-FASVSNMMS-MALLRRIEHPTAGNTEAWEDT }\end{array}$

\section{C}

c.445C $>\mathrm{T}$

p.P152L

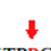

Homo

AIYKTPPGIQKSAVA

AIYKTPPGIQKSAVA

$\begin{array}{ll}\text { Mus } & \text { Rattus }\end{array}$

Xenopus PLFKTPPGLQKAPVP

Macaca AIYKTPPGIQKSAVA

Gallus AIYKTPPGIQKNSVP

Fig.1.Pedigrees with Neurofibromatosis 1 (NF1)-Congenital pseudarthrosis of the tibia (CPT) and putative pathogenic mutations

(A) Each proband is indicated by an arrow. The diagonal line indicates a deceased family member. The family members enrolled in this study are marked with asterisks. Segregation of mutations 
with Sanger sequencing is represented by arrows. (B) Some conservation of the protein residues is targeted by the mutation identified in NF1 patients. (C) Protein sequence alignment of $B C O R$ orthologs shows the region surrounding the mutated P152L.
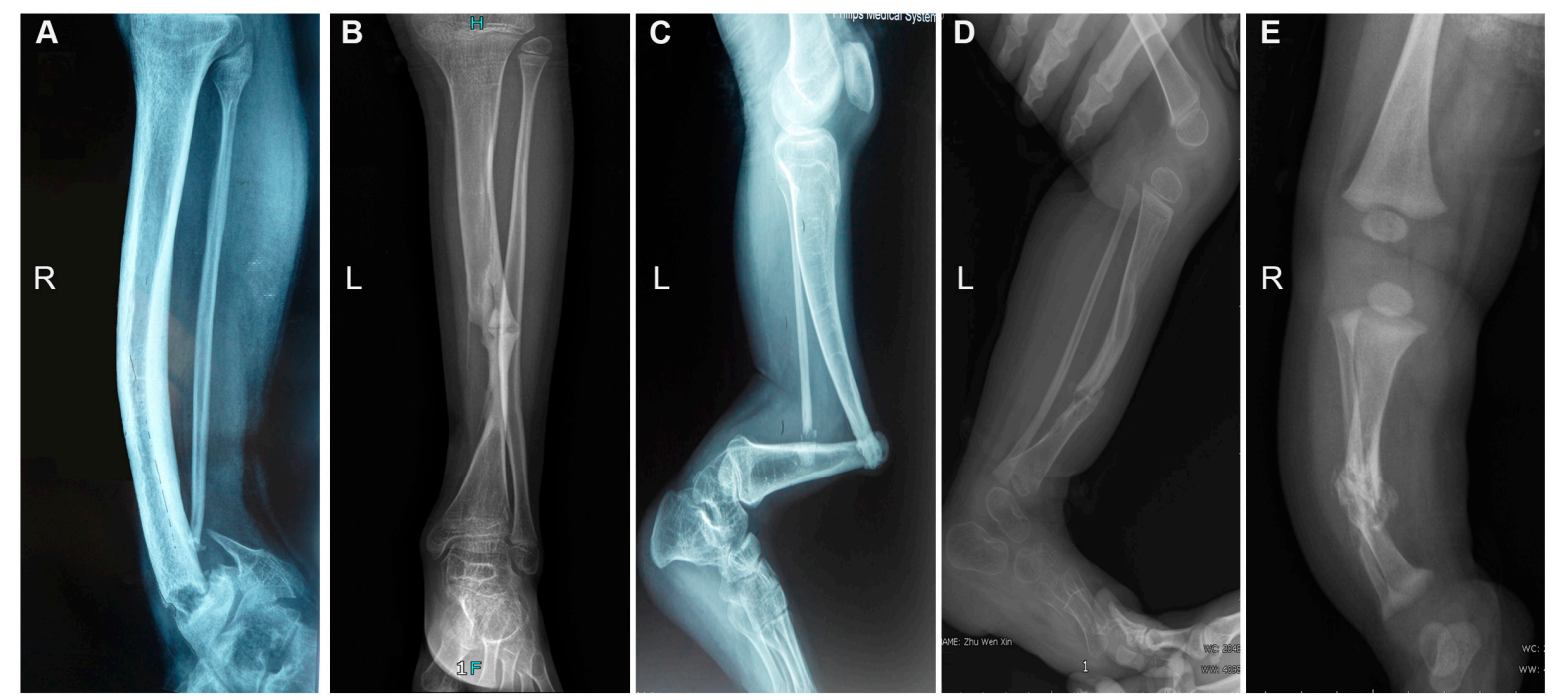

Fig.2. Radiographs of five probands showing Congenital pseudarthrosis of the tibia (CPT), just before surgical intervention.

(A) Lateral radiograph of right leg (the patient A1-III:1) ; (B) Anteroposterior radiograph of left leg (the patient A2-II:1); (C) Lateral radiograph of left leg (the patient A3-II:2); (D) Lateral radiograph of left leg (the patient A4-II:1); (E) Lateral radiograph of right leg (the patient A5-II:1) 
Table 1. Clinical features of each CPT patient with NF1 and summary of mutations detected in the NF1 Gene

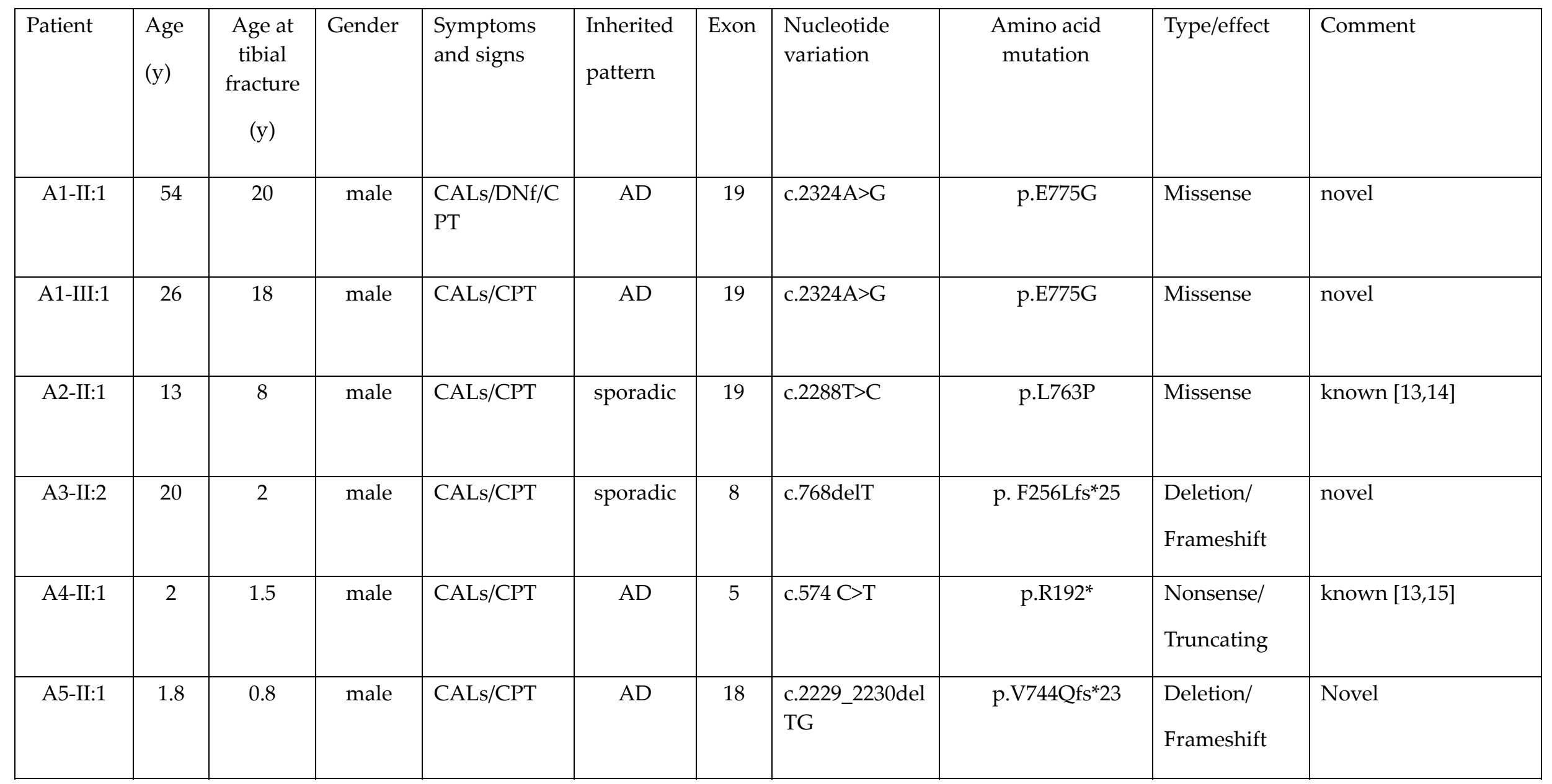

CALs: Cafè-au-Lait Spots, DNf: Dermal Neurofibromas, CPT: congenital pseudarthrosis of the tibia, AD: autosomal dominant 
Table 2. Overview of data generation

\begin{tabular}{|c|c|c|c|c|c|c|}
\hline Patient & A1-II:1 & A1-III:1 & A2-II:1 & A3-II:2 & A4-II:1 & A5-II:1 \\
\hline Targeted region & Whole exome & Whole exome & $N F 1, N F 2$ & $N F 1, N F 2$ & $N F 1, N F 2$ & $N F 1, N F 2$ \\
\hline Raw reads & 146079974 & 165561368 & 28663620 & 39678421 & 28694073 & 34674385 \\
\hline Reads mapped to targeted region & 107280276 & 119981218 & 19459821 & 29768932 & 19438762 & 20984769 \\
\hline Coverage of targeted region (\%) & 99.6 & 99.6 & 100.00 & 100.00 & 100.00 & 99.59 \\
\hline Mean depth of targeted region & 65.38 & 67.88 & 198.17 & 643.08 & 178.71 & 228.77 \\
\hline Depth of targeted region $4 \mathrm{X}(\%)$ & 98.6 & 98.5 & 100.00 & 100.00 & 100.00 & 99.56 \\
\hline Depth of targeted region 10X (\%) & 96.9 & 96.7 & 98.9 & 100.00 & 98.3 & 97.8 \\
\hline Depth of targeted region 30X (\%) & 92.1 & 92.0 & 96.11 & 99.94 & 95.41 & 91.27 \\
\hline
\end{tabular}


Acknowledgments: This study was supported by the National Basic Research Program (973 Program) (No. 2011CB707800, awarded to Lu Shen), the National Natural Science Foundation of China (No. 81171068 and No. 81471295, awarded to Lu Shen), and the Hunan Provincial Science \& Technology Department Program (No.2012SK3197, awarded to Zhangyuan Lin). We are grateful to all the subjects for participation in our study.

Author contributions: L. S, Z.L conceived and designed the experiments; Z.Z, Y.Z, X.W, F.L, X.L, L.Z, L.H performed the experiments; Z.Z, L. S, Z.L wrote the paper; Z.Z, Y.Z, X.W, F.L, L.S, Z.L provided study materials or patients; S.S contributed reagents/materials/analysis tools.

Conflicts of Interests: The authors declare no conflict of interest.

\section{References}

1. Williams, V. C.; Lucas, J.; Babcock, M. A.; Gutmann, D. H.; Korf, B.; Maria, B. L., Neurofibromatosis type 1 revisited. Pediatrics 2009, 123, (1), 124-33.

2. Shen, M. H.; Harper Ps Fau - Upadhyaya, M.; Upadhyaya, M., Molecular genetics of neurofibromatosis type 1 (NF1). Journal of medical genetics 1996, 33(1), (0022-2593 (Print)), 2-17.

3. Friedman, J. M.; Birch, P. H., Type 1 neurofibromatosis: a descriptive analysis of the disorder in 1,728 patients. American journal of medical genetics 1997, 70, (2), 138-43.

4. Crawford, A. H.; Schorry, E. K., Neurofibromatosis in children: the role of the orthopaedist. The Journal of the American Academy of Orthopaedic Surgeons 1999, 7, (4), 217-30.

5. Cho, T. J.; Seo, J. B.; Lee, H. R.; Yoo, W. J.; Chung, C. Y.; Choi, I. H., Biologic characteristics of fibrous hamartoma from congenital pseudarthrosis of the tibia associated with neurofibromatosis type 1 . The Journal of bone and joint surgery. American volume 2008, 90, (12), 2735-44.

6. Hermanns-Sachweh, B.; Senderek, J.; Alfer, J.; Klosterhalfen, B.; Buttner, R.; Fuzesi, L.; Weber, M., Vascular changes in the periosteum of congenital pseudarthrosis of the tibia. Pathology, research and practice 2005, 201, (4), 305-12.

7. Hefti, F.; Bollini, G.; Dungl, P.; Fixsen, J.; Grill, F.; Ippolito, E.; Romanus, B.; Tudisco, C.; Wientroub, S., Congenital pseudarthrosis of the tibia: history, etiology, classification, and epidemiologic data. Journal of pediatric orthopedics. Part B 2000, 9, (1), 11-5.

8. Leskela, H. V.; Kuorilehto, T.; Risteli, J.; Koivunen, J.; Nissinen, M.; Peltonen, S.; Kinnunen, P.; Messiaen, L.; Lehenkari, P.; Peltonen, J., Congenital pseudarthrosis of neurofibromatosis type 1: impaired osteoblast differentiation and function and altered NF1 gene expression. Bone 2009, 44, (2), 243-50.

9. Ippolito, E.; Corsi, A.; Grill, F.; Wientroub, S.; Bianco, P., Pathology of bone lesions associated with congenital pseudarthrosis of the leg. Journal of pediatric orthopedics. Part B 2000, 9, (1), 3-10.

10. Kjell, V. R.; Hilde, B.; Eric, L.; Johan, L.; Armand, L., Prevalence of neurofibromatosis type 1 in congenital pseudarthrosis of the tibia. European journal of pediatrics 2016.

11. Stevenson, D. A.; Zhou, H.; Ashrafi, S.; Messiaen, L. M.; Carey, J. C.; D'Astous, J. L.; Santora, S. D.; Viskochil, D. H., Double inactivation of NF1 in tibial pseudarthrosis. American journal of human genetics 2006, 79, (1), 143-8.

12. Sant, D. W.; Margraf, R. L.; Stevenson, D. A.; Grossmann, A. H.; Viskochil, D. H.; Hanson, H.; Everitt, M. D.; Rios, J. J.; Elefteriou, F.; Hennessey, T.; Mao, R., Evaluation of somatic mutations in tibial pseudarthrosis samples in neurofibromatosis type 1. Journal of medical genetics 2015, 52, (4), 256-61.

13. Fahsold, R.; Hoffmeyer, S.; Mischung, C.; Gille, C.; Ehlers, C.; Kucukceylan, N.; Abdel-Nour, M.; Gewies, A.; Peters, H.; Kaufmann, D.; Buske, A.; Tinschert, S.; Nurnberg, P., Minor lesion mutational spectrum of the entire NF1 gene does not explain its high mutability but points to 
a functional domain upstream of the GAP-related domain. American journal of human genetics 2000, 66, (3), 790-818.

14. Valero, M. C.; Martin, Y.; Hernandez-Imaz, E.; Marina Hernandez, A.; Melean, G.; Valero, A. M.; Javier Rodriguez-Alvarez, F.; Telleria, D.; Hernandez-Chico, C., A highly sensitive genetic protocol to detect NF1 mutations. The Journal of molecular diagnostics : JMD 2011, 13, (2), 113-22.

15. De Luca, A.; Schirinzi, A.; Buccino, A.; Bottillo, I.; Sinibaldi, L.; Torrente, I.; Ciavarella, A.; Dottorini, T.; Porciello, R.; Giustini, S.; Calvieri, S.; Dallapiccola, B., Novel and recurrent mutations in the NF1 gene in Italian patients with neurofibromatosis type 1. Human mutation 2004, 23, (6), 629.

16. Paria, N.; Cho, T. J.; Choi, I. H.; Kamiya, N.; Kayembe, K.; Mao, R.; Margraf, R. L.; Obermosser, G.; Oxendine, I.; Sant, D. W.; Song, M. H.; Stevenson, D. A.; Viskochil, D. H.; Wise, C. A.; Kim, H. K.; Rios, J. J., Neurofibromin deficiency-associated transcriptional dysregulation suggests a novel therapy for tibial pseudoarthrosis in NF1. Journal of bone and mineral research : the official journal of the American Society for Bone and Mineral Research 2014, 29, (12), 2636-42.

17. Viskochil, D., In search of the Holy Grail: NF1 mutation analysis and genotype-phenotype correlation. Genetics in medicine : official journal of the American College of Medical Genetics 1999, $1,(6), 245-7$.

18. Stevenson, D. A.; Birch, P. H.; Friedman, J. M.; Viskochil, D. H.; Balestrazzi, P.; Boni, S.; Buske, A.; Korf, B. R.; Niimura, M.; Pivnick, E. K.; Schorry, E. K.; Short, M. P.; Tenconi, R.; Tonsgard, J. H.; Carey, J. C., Descriptive analysis of tibial pseudarthrosis in patients with neurofibromatosis 1. American journal of medical genetics 1999, 84, (5), 413-9.

19. Nagase, T.; Kikuno, R.; Nakayama, M.; Hirosawa, M.; Ohara, O., Prediction of the coding sequences of unidentified human genes. XVIII. The complete sequences of 100 new cDNA clones from brain which code for large proteins in vitro. DNA research: an international journal for rapid publication of reports on genes and genomes 2000, 7, (4), 273-81.

20. Huynh, K. D.; Fischle, W.; Verdin, E.; Bardwell, V. J., BCoR, a novel corepressor involved in BCL-6 repression. Genes $\mathcal{E}$ development 2000, 14, (14), 1810-23.

21. Fan, Z.; Yamaza, T.; Lee, J. S.; Yu, J.; Wang, S.; Fan, G.; Shi, S.; Wang, C. Y., BCOR regulates mesenchymal stem cell function by epigenetic mechanisms. Nature cell biology 2009, 11, (8), 1002-9.

22. Du, J.; Ma, Y.; Ma, P.; Wang, S.; Fan, Z., Demethylation of epiregulin gene by histone demethylase FBXL11 and BCL6 corepressor inhibits osteo/dentinogenic differentiation. Stem cells (Dayton, Ohio) 2013, 31, (1), 126-36.

23. Ng, D.; Thakker, N.; Corcoran, C. M.; Donnai, D.; Perveen, R.; Schneider, A.; Hadley, D. W.; Tifft, C.; Zhang, L.; Wilkie, A. O.; van der Smagt, J. J.; Gorlin, R. J.; Burgess, S. M.; Bardwell, V. J.; Black, G. C.; Biesecker, L. G., Oculofaciocardiodental and Lenz microphthalmia syndromes result from distinct classes of mutations in BCOR. Nature genetics 2004, 36, (4), 411-6.

24. Neurofibromatosis. Conference statement. National Institutes of Health Consensus Development Conference. Archives of neurology 1988, 45, (5), 575-8.

25. Li, H.; Durbin, R., Fast and accurate short read alignment with Burrows-Wheeler transform. Bioinformatics (Oxford, England) 2009, 25, (14), 1754-60.

26. Danecek, P.; Auton, A.; Abecasis, G.; Albers, C. A.; Banks, E.; DePristo, M. A.; Handsaker, R. E.; Lunter, G.; Marth, G. T.; Sherry, S. T.; McVean, G.; Durbin, R., The variant call format and VCFtools. Bioinformatics (Oxford, England) 2011, 27, (15), 2156-8.

27. Robinson, J. T.; Thorvaldsdottir, H.; Winckler, W.; Guttman, M.; Lander, E. S.; Getz, G.; Mesirov, J. P., Integrative genomics viewer. Nature biotechnology 2011, 29, (1), 24-6.

(C) 2016 by the authors; licensee Preprints, Basel, Switzerland. This article is an open access article distributed under the terms and conditions of the Creative Commons by Attribution (CC-BY) license (http://creativecommons.org/licenses/by/4.0/). 